\title{
A NEW LOOK AT EXOTIC DECAYS: $\tilde{\rho}\left(1^{-+}, I=1\right) \rightarrow \eta^{\prime} \pi$ VERSUS $\rho \pi$
}

\author{
J.-M. FRÈRE ${ }^{1,2,3}$ and S. TITARD \\ Randall Laboratory of Physics, Department of Physics, University of Michigan, Ann Arbor, MI 48109-1120, USA
}

Received 29 August 1988

\begin{abstract}
We study the decays of the exotic meson $\tilde{\rho}\left(1^{-+}, I=1\right)$ into $\eta^{\prime} \pi$ and $\eta \pi$. We assume hybrid quark-glue structure and exhibit a large contribution to this decay in the QCD sum rules approach, and suggest that $\eta^{\prime} \pi$ rather than $\rho \pi$ might be the dominant decay channel.
\end{abstract}

Exotic mesons, in particular hybrid (valence gluon + quarks) states have recently received renewed attention, both from the experimental and theoretical sides. Central to that interest are the apparently conflicting data dealing with the $J^{P C} I=1^{-+} 1$ state often named $\tilde{\rho}$. Ref. [1] reports a signal at 1400 $\mathrm{MeV}$ in a partial wave analysis of $\pi^{-} \mathbf{p} \rightarrow \pi^{0} \eta^{0}+$ neutrals, which could be interpreted in terms of $\tilde{\rho} \rightarrow$ $\eta \pi$, with an unspecified production mechanism. Due to its focus on neutrals, this experiment is blind to the $\rho \pi$ channel. Ref. [2], on the other hand, bases itself on the widely held theoretical prejudice that $\tilde{\rho} \rightarrow \rho \pi$ is by far the dominant decay channel. Since $\tilde{\rho} \rightarrow \gamma \pi$ is directly related to $\tilde{\rho} \rightarrow \rho \pi$ by vector dominance considerations, they can, by consideration of the Primakoff production in $\pi$-nucleus scattering put the strict bound $\Gamma(\tilde{\rho} \rightarrow \rho \pi)<20 \mathrm{MeV}$ for $m_{\tilde{\rho}}<$ $1.5 \mathrm{GeV}$.

The two results conflict with the usually expected values of

$\Gamma(\tilde{\rho} \rightarrow \rho \pi) \gg \Gamma\left(\tilde{\rho} \rightarrow \eta^{\prime} \pi\right)>\Gamma(\tilde{\rho} \rightarrow \eta \pi)$.

This situation prompted us to reconsider the arguments leading to (1), more specifically in the context of QCD sum rules which seem to provide the strongest statements in that domain. We conclude that $\rho^{\prime} \pi$ is most likely the dominant decay mode.

We thus focus on $\widetilde{\rho}^{0} \rightarrow \eta^{\prime} \pi^{0}$ and take the following choices for the currents associated with $\eta^{\prime}, \eta, \pi^{0}, \tilde{\rho}$

1 Chercheur qualifié du FNRS.

2 Physique Théorique CP225, ULB-Campus Plaine, B-1050 Brussels, Belgium.

3 Work supported in part by NATO. (see ref. [3]; $\theta$ is found to obey: $-20^{\circ} \leqslant \theta \leqslant-16^{\circ}$ )

$$
\begin{aligned}
& \phi_{\eta}=\frac{\partial_{\mu} A_{8}^{\mu}}{f_{8}} \frac{\cos \theta}{m_{\eta}^{2}}-\frac{\partial_{\mu} A_{0}^{\mu}}{f_{0}} \frac{\sin \theta}{m_{\eta}^{2}}, \\
& \phi_{\eta^{\prime}}=\frac{\partial_{\mu} A_{8}^{\mu}}{f_{8}} \frac{\sin \theta}{m_{\eta^{\prime}}^{2}}+\frac{\partial_{\mu} A_{0}^{\mu}}{f_{0}} \frac{\cos \theta}{m_{\eta^{\prime}}^{2}}, \\
& \phi_{\pi^{0}}=\frac{\partial_{\mu} A_{3}^{\mu}}{f_{\pi}} \frac{1}{m_{\pi^{2}}},
\end{aligned}
$$

where

$$
\begin{aligned}
& A_{0}^{\mu}=\left(\mathrm{u}_{5}^{\mu}+\mathrm{d}_{5}^{\mu}+\mathrm{s}_{5}^{\mu}\right) / \sqrt{6}, \\
& A_{8}^{\mu}=\left(\mathrm{u}_{5}^{\mu}+\mathrm{d}_{5}^{\mu}-2 \mathrm{~s}_{5}^{\mu}\right) /(2 \sqrt{3}), \\
& A_{3}^{\mu}=\left(\mathrm{u}_{5}^{\mu}+\mathrm{d}_{5}^{\mu}\right) / 2, \\
& \mathrm{q}_{5}^{\mu} \equiv \overline{\mathrm{q}} \gamma^{\mu} \gamma_{5} \mathrm{q},
\end{aligned}
$$

with $f_{\pi}=92 \mathrm{MeV}$

$\phi_{\rho_{0}}^{\mu}=g_{\mathrm{s}} G_{a}^{\mu \nu} \frac{1}{2}\left(\overline{\mathbf{u}} \gamma_{\nu} \gamma_{5} \lambda^{a} \mathrm{u}-\overline{\mathrm{d}} \gamma_{\nu} \gamma_{5} \lambda^{a} \mathrm{~d}\right) / f_{\tilde{\rho}} m_{\bar{\rho}}^{3}$,

where $g_{\mathrm{s}}$ is the strong coupling constant, and $G_{a}^{\mu \nu}$ is the gluon field strength. It was noted in ref. [4] that the quark part of the divergences (2) and (3) does not contribute to the decays in leading order; this statement was further substantiated in ref. [5].

The important connection of $\eta$ and $\eta^{\prime}$ to two-gluon states through the anomaly must however be kept in mind, and the graph presented in fig. 1 was first brought to attention in ref. [6]. Here the $\eta^{\prime}$-twogluon vertex is due to the anomalous divergence

$\partial_{\mu} A_{0}^{\mu}=\frac{1}{\sqrt{6}} \frac{3 \alpha_{\mathrm{s}}}{4 \pi} G^{\mu \nu} \tilde{G}_{\mu \nu}+$ mass terms . 


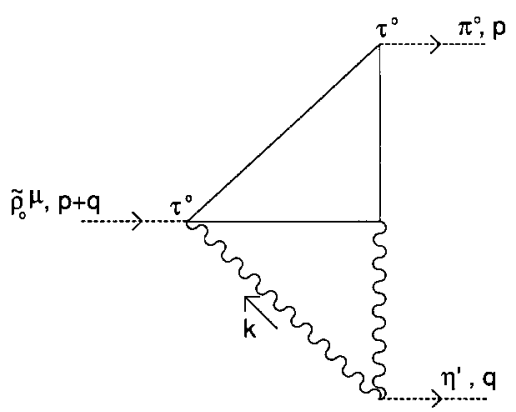

Fig. 1. Anomalous contribution to $\tilde{\rho} \rightarrow \eta^{\prime} \pi$. Here $\tau^{0}$ stands for $\tau^{3}$; similar contributions exist for the charged particles with $\tau^{0}$ replaced by $\tau^{ \pm}$

In the context of a QCD sum rules approach the authors then proceeded to evaluate the various diagrams derived from this by the insertions of condensates $\langle\bar{\psi} \psi\rangle,\langle\bar{\psi} \mathrm{G} \psi\rangle, \ldots$. It strikes us however that the bare diagram by itself might bring the dominant contribution, due again to the presence of an anomaly in the remaining fermion triangle. It would be wrong indeed in the present context to replace $\partial_{\mu} A_{3}^{\mu}$ by the usual $m_{\mathrm{u}} \overline{\mathrm{u}} \gamma_{5} \mathrm{u}+\ldots$ term, since the $\tau$ matrices at the vertices conjure to make the graph anomalous.

From this it becomes straightforward to evaluate this new contribution; we simply provide the salient points. The only difficulty stems from the ultraviolet divergence of the graph, which results when the fermionic triangle is shrunk, and reduces to $\epsilon_{p \rho \sigma \tau}(p+$ $q+k)^{\rho}(k+q)^{\tau}$. Our only resource consists in the introduction of a cut-off, which in some way represents the wave function of the $\tilde{\rho}$ particle. It is quite obvious on physical grounds that such a momentum dependence has to occur due to the composite nature of $\tilde{\rho}$, but it is a lot harder to specify its shape. In order to keep things simple, we simply introduce a factor $\left[A^{2} /\left(\left|k^{2}\right|+A^{2}\right)\right]^{2}$ in the left-over integral. Let us define

$$
\begin{gathered}
\left\langle 0\left|\mathrm{~T} \phi_{\eta^{\prime}}(q) \phi_{\pi^{0}}(p) \phi_{\rho_{0}}^{\mu}(p+q)\right| 0\right\rangle \\
=\left(p^{\mu}-q^{\mu}\right) A+\left(p^{\mu}+q^{\mu}\right) A^{\prime} .
\end{gathered}
$$

We get:

$$
\begin{aligned}
|A| & =\alpha_{\mathrm{s}}^{2} \frac{1}{(2 \pi)^{4}} \frac{24}{\sqrt{6}} \frac{A^{2}}{2} \\
& \times\left|q^{2}\left(2 K_{1}-K_{2}\right)+p q\left(2 K_{1}-4 K_{2}\right)\right| \cos \theta .
\end{aligned}
$$

We are only interested in $A$ since the $A^{\prime}$ contribution is orthogonal to the $\tilde{\rho}$ spin in its rest frame and thus does not contribute. $K_{1}$ and $K_{2}$ are given by

$$
\begin{aligned}
& K_{1}=\int_{0}^{1} \mathrm{~d} z \int_{0}^{1} \mathrm{~d} y(1-z) y\left[K z+(1-z)-K z^{2} y\right]^{-1}, \\
& K_{2}=\int_{0}^{1} \mathrm{~d} z \int_{0}^{1} \mathrm{~d} y\left(z-z^{2}\right) y^{2}\left[K^{2}+(1-z)-K z^{2} y\right]^{-1}, \\
& K=Q^{2} / \Lambda^{2} .
\end{aligned}
$$

We choose the unphysical point $q^{2}=-Q^{2}=p^{2}=$ $(p+q)^{2}$. We have not attempted a complete analytical evaluation of these functions; instead, we present in fig. 2 a plot of $K_{1}$ and $K_{2}$ together with a power fit to their dependence in $K$. We have now to compare the value of (9) to the phenomenological approach, continued to unphysical $q^{2}$

$A_{\mathrm{ph} .}=g_{\tilde{\rho} \mathfrak{\eta}^{\prime} \pi} \frac{f_{\pi} m_{\pi}^{2}}{Q^{2}+m_{\pi}^{2}} \frac{f_{\tilde{\rho}} m_{\tilde{\rho}}^{2}}{Q^{2}+m_{\tilde{\rho}}^{2}} \frac{f_{\eta^{\prime}} m_{\eta_{\gamma^{\prime}}}^{2}}{Q^{2}+m_{\eta^{\prime}}^{2}}$.

Dependence of $\left(K_{1}+K_{2}\right)$ on $Q^{2} / \Lambda^{2}$

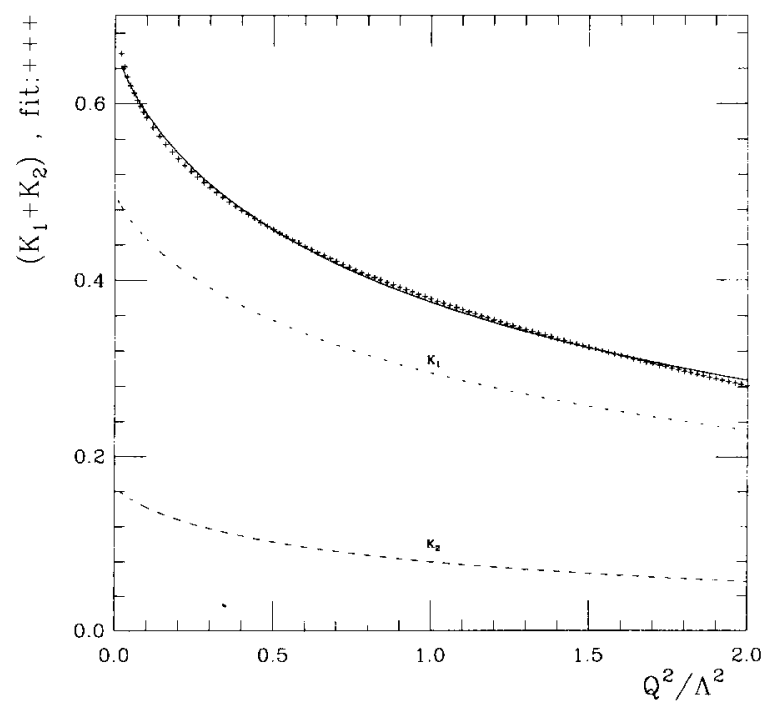

Fig. 2. $K_{1}, K_{2}$ and fit to $K_{1}+K_{2}$. 
The comparison of (9) and (11) is pretty difficult, since the momentum dependence of both expressions is so different. Our main point is that the contribution (9) is large when compared to the terms retained in ref. [6], the latter being suppressed by at least one power of $\left(m_{\mathrm{d}}+m_{\mathrm{u}}\right)$.

In order to make the comparison more striking, we will follow closely the steps taken in ref. [6] (see however our reservations below). For this purpose, we neglect $m_{\pi}^{2}$ in the denominator of (11) and multiply both (9) and (11) by $Q^{2}$ before taking the inverse Laplace transform, i.e. applying the operation [7]

$$
L=\lim _{\substack{Q^{2}, n \rightarrow \infty \\ Q^{2} / n=1 / \tau}} \frac{1}{(n-1) !}\left(Q^{2}\right)^{n}\left(\frac{-\mathrm{d}}{\mathrm{d} Q^{2}}\right)^{n} .
$$

The fit presented in fig. 2 corresponds to

$K_{1}+K_{2}=a^{\prime}\left(\frac{Q^{2}}{A^{2}}\right)^{a}-b^{\prime}\left(\frac{Q^{2}}{A^{2}}\right)^{b}$,

and gives $a=0.017, b=0.28, a^{\prime}=0.88, b^{\prime}=0.5$. Unfortunately it becomes negative for large $Q^{2}$. We have checked that more extended (but less accurate) fits give qualitatively similar results (in particular $c^{\prime}\left(Q^{2}\right)$ $\left.A^{2}\right)^{-c}$ whose divergence at $Q^{2}=0$ is anyway suppressed by the extra powers of $Q^{2}$ in (9)). The comparison of the transforms of (9) and (11) yields:

$$
\begin{aligned}
& g_{\tilde{\rho} \eta^{\prime} \pi}=\frac{\cos \theta}{(2 \pi)^{4}} \frac{24}{\sqrt{6}} \frac{1}{2} \frac{m_{\tilde{\rho}}^{2}-m_{\eta^{\prime}}^{2}}{f_{0} m_{\eta^{\prime}}^{2} f_{\pi} m_{\pi}^{2} f_{\tilde{\rho}} m_{\tilde{\rho}}^{2}} \\
& \times\left. G_{\eta^{\prime}}(A, \tau)\right|_{\min } A^{2},
\end{aligned}
$$

with

$$
\begin{gathered}
G_{\eta^{\prime}}(\Lambda, \tau)=a^{\prime} G_{\eta^{\prime}}^{a}\left(\Lambda^{2}\right)^{-a}[\Gamma(-2-a)]^{-1} \\
-b^{\prime} G_{\eta^{\prime}}^{b}\left(A^{2}\right)^{-b}[\Gamma(-2-b)]^{-1},
\end{gathered}
$$

where

$$
\begin{aligned}
& G_{\mathrm{\eta}^{\prime}}^{a}(\tau)=\tau^{-3-a} \alpha_{\mathrm{s}}^{2}(\tau) \\
& \quad \times\left[\exp \left(-m_{\eta^{\prime}}^{2} \tau\right)-\exp \left(-m_{\tilde{\mathrm{\rho}}}^{2} \tau\right)\right]^{-1},
\end{aligned}
$$

where $\alpha_{\mathrm{s}}(\tau)$ is the strong coupling constant with $Q^{2}$ replaced by $1 / \tau$. The functions $G_{\eta}(\tau)$ and $G_{\eta^{\prime}}(\tau)$ are plotted in fig. 3 ; we follow the usual prescription in choosing to evaluate $g_{\tilde{\rho} \eta^{\prime} \pi}$ for the value of $\tau$ corre-
Functions $G_{\eta}(\tau)$ and $G_{\eta^{\prime}}(\tau)$

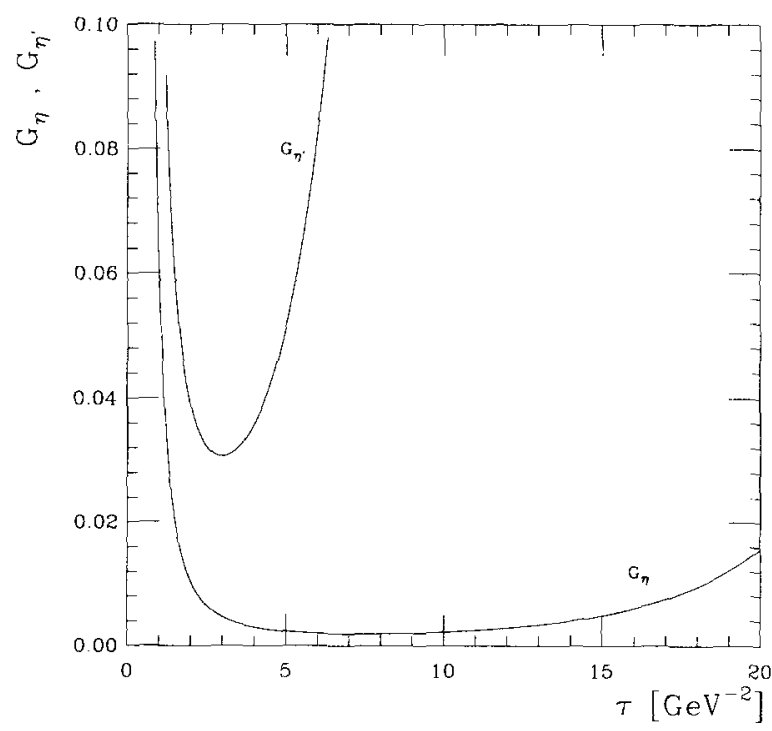

Fig. 3. $G_{\eta}, G_{\eta^{\prime}}(\tau)$ evaluated for $m_{\bar{\rho}}=1.4 \mathrm{GeV} ; A=1 \mathrm{GeV}$. Similar curves are obtained for the other values of the parameters quoted in the text.

sponding to stationarity of $G(\tau)$. Of course, our result is very sensitive to the value of the cut-off. Since $A$ is supposed to represent the physical cut-off in the $\tilde{\rho}$ wave function we could choose somewhat arbitrarily $A=m_{\tilde{\rho}}=1.4 \mathrm{GeV}$; using $f_{\tilde{\rho}}=0.02 \mathrm{GeV}$ from ref. [6] we get:

$g_{\tilde{\rho} \eta^{\prime} \pi}=16.5$,

which according to

$\Gamma\left(\tilde{\rho} \rightarrow \eta^{\prime} \pi\right) \simeq\left(g_{\hat{\rho} \eta^{\prime} \pi}\right)^{2} \frac{m_{\hat{\rho}}}{48 \pi}\left(1-\frac{m_{\eta^{\prime}}^{2}}{m_{\hat{\rho}}^{2}}\right)^{3}$

would lead to a width of $380 \mathrm{MeV}$. For $A=m_{\tilde{\rho}}=$ $1.6 \mathrm{GeV}$, as considered in ref. [6] we obtained $\Gamma \sim 1 \mathrm{GeV}$ !

Clearly this approximation is not reliable, and for various reasons:

- we are actually trying to compare two wildly different functions. (9) decreases with $Q^{2}$ while (11) increases with $Q^{2}$;

- the inverse Laplace transform acts in a capricious way on positive powers with vanishing results for all integral powers. Its main justification in this context stems however from the remarkable successes met in the case of ordinary mesons [8]; 
- the phenomenological approximation may be at fault; for instance, $g_{\overline{\bar{p}^{\prime}} \boldsymbol{} \pi}$ is presumably a function of $Q^{2}$, the physical process could then be considerably reduced in view of the little energy available for the decay;

- our choice of the cut-off mass is quite arbitrary; for instance, taking $A=1 \mathrm{GeV}$ yields

$\Gamma\left(\tilde{\rho} \rightarrow \eta^{\prime} \pi\right)=150 \mathrm{GeV}$.

We do not want to insist further on the possible difficulties of the approach, since we think our main point is now taken. Namely, the presence of anomalies induce a large contribution to $\tilde{\rho} \rightarrow \eta^{\prime} \pi$ and subsequently to $\tilde{\rho} \rightarrow \eta \pi$.

While (2) and (3) would lead us to expect $g_{\hat{p} \eta \pi} /$ $g_{\tilde{\rho} \eta^{\prime} \pi}=\operatorname{tg} \theta$, the QCD sum rules approach seems more sensitive however to SU (3) breaking and would yield the following results

$\frac{g_{\tilde{\rho} \eta \pi}}{g_{\tilde{\rho} \eta^{\prime} \pi}}=\operatorname{tg} \theta \frac{\left.G_{\eta}(\tau, \Lambda)\right|_{\min }}{\left.G_{\eta^{\prime}}(\tau, \Lambda)\right|_{\min }} \frac{m_{\eta^{\prime}}^{2}\left(m_{\tilde{\rho}}^{2}-m_{\eta}^{2}\right)}{m_{\eta}^{2}\left(m_{\tilde{\rho}}^{2}-m_{\eta^{\prime}}^{2}\right)}$.

Note that this ratio is nearly independent of the cutoff $A$. We obtain for $m_{\tilde{\rho}}=1.4 \mathrm{GeV}$

$\frac{g_{\tilde{\rho} \eta \pi}}{g_{\tilde{\rho} \eta^{\prime} \pi}}=0.31 \operatorname{tg} \theta, \frac{\Gamma(\tilde{\rho} \rightarrow \eta \pi)}{\Gamma\left(\tilde{\rho} \rightarrow \eta^{\prime} \pi\right)}=3.5 \%$.

Irrespective of the details of the above treatment, we conclude that an anomalous contribution to $\tilde{\rho} \rightarrow$ $\eta^{\prime} \pi$ is large (eq. (9)) while no similar contribution arises for $\tilde{\rho} \rightarrow \rho \pi, \tilde{\rho} \rightarrow \gamma \pi$. While impressed by the size of the new term, we have stressed the difficulties inherent in its interpretation. It is our belief that we have sufficient evidence to suggest that the $\tilde{\rho} \rightarrow \eta^{\prime} \pi$ and $\tilde{\rho} \rightarrow \eta \pi$ transitions are largely enhanced with respect to the previous estimates, and that $\eta^{\prime} \pi$ might be the dominant decay mode. On the other hand, the data of ref. [2] still seem incompatible with the width of $\tilde{\rho} \rightarrow \rho \pi$ and $\tilde{\rho} \rightarrow \gamma \pi$ estimated in ref. [6]. In view of the suspected difficulties in the use of Laplace transforms, we think it wise to reserve judgement, and hope our experimentalist colleagues will succeed in measuring the decays into vectors and pseudoscalars in the same set up.

We want also to stress that there is no disagreement with the work of ref. [5], which indeed states that the main contribution should come from the Zweig-suppressed graphs considered here. We have no comment on other approaches.

J.-M. Frère wants to thank the Particle Theory group, University of Michigan, Ann Arbor for its constant support; in particular numerous discussions with R. Akhoury, G. Kane, M. Leurer, Y.-P. Yao. Thanks also to J.P. Stroot, F. Binon and the NA12 group. S. Titard thanks R. Bouamrane for his help.

\section{References}

[1] D. Alde et al., Phys. Lett. B 205 (1988) 397.

[2] M. Zielinski et al., Z. Phys. C 31 (1988) 345.

[3] R. Akhoury and J.-M. Frère, $\eta-\eta^{\prime}$ mixing and anomalies, University of Michigan preprint UM-TH-87-19, Phys. Lett. B., to be published.

[4] F. de Viron and J. Govaerts, Phys. Rev. Lett. 53 (1984) 2207.

[5] R. Iddir, A. Le Yaouanc, L. Oliver, O. Pene and J.C. Raynal, Selection rule for $1^{-+}$decay into $\eta \pi$ from QCD, preprint LPTHE Orsay 88/80.

[6] J.L. LaTorre, P. Pascual and S. Narison, Z. Phys. C 34 (1987) 347 , and references therein.

[7] M.A. Shifman, A.I. Vainshtein and V.I. Zakharov, Nucl. Phys. B 147 (1979) 385.

[8] S. Narison and N. Paver, Z. Phys. C 22 (1984) 69. 